\title{
س
}

$>\mathrm{DE}$

$\stackrel{1}{\simeq}$ PÉDAGOGIE

Recherches en éducation

175 | avril-juin 2011

Penser les choix scolaires

\section{Quels choix ? Pour quelle école?}

\section{Françoise Euvrard}

\section{(2) OpenEdition}

\section{Journals}

Édition électronique

URL : http://journals.openedition.org/rfp/3061

DOI : 10.4000/rfp.3061

ISSN : 2105-2913

Éditeur

ENS Éditions

Édition imprimée

Date de publication : 1 juin 2011

Pagination : 73-76

ISBN : 978-2-84788-320-6

ISSN : 0556-7807

Référence électronique

Françoise EFuvrard, «Quels choix ? Pour quelle école? », Revue française de pédagogie [En ligne], 175 | avril-juin 2011, mis en ligne le 15 juin 2015, consulté le 30 avril 2019. URL : http:// journals.openedition.org/rfp/3061; DOl : 10.4000/rfp.3061 


\section{Contrepoints}

\section{Quels choix? Pour quelle école?}

\section{Françoise OEuvrard}

Si les recherches sur la construction des choix scolaires demeurent pertinentes, elles le sont en partie parce qu'elles mettent au jour l'interaction des facteurs en jeu dans les processus de formation des inégalités sociales à l'école, en montrant en particulier le caractère déterminant des inégalités d'accès aux savoirs scolaires. Aussi les articles rassemblés dans ce dossier, comme le disent explicitement Sylvain Broccolichi et Rémi Sinthon, invitent les chercheurs à développer des travaux sur la relation entre contextes sociaux et transmission des savoirs. Nombre de travaux pointent l'importance de l'échec scolaire en France, son évolution alarmante et son inscription spatiale alors que s'accumulent, dans le système éducatif, des dispositifs ou politiques scolaires qui, loin de relever ce défi posé par les inégalités sociales de réussite, visent plutôt à " libéraliser l'offre scolaire ${ }^{1}$ ", avec des conséquences qui peuvent renforcer les disparités initiales. Nous souhaiterions ici questionner le contexte social et scolaire dans lequel ces politiques (suppression de la carte scolaire, augmentation du rôle des familles dans les choix scolaires, mise en place de l'orientation active...) sont instaurées.

Tout d'abord, l'échec scolaire est encore précoce et toujours cumulatif. Même si la pratique du redoublement a fortement diminué, avec la mise en place des cycles à l'école élémentaire et des classes à double niveau, et si la baisse des redoublements est particulièrement sensible au cours préparatoire
(CP), les études statistiques montrent que les écarts de réussite au cours des premières années de scolarité restent très marqués : les enfants qui entrent à l'école ont une distance à la culture légitime plus ou moins grande selon leur milieu social et ils vont connaître, au cours de leur scolarité, des progressions différentielles suivant leur origine sociale, même si l'on tient compte de leur niveau initial, si bien que les écarts se creusent au cours de la scolarité élémentaire (Caille \& Rosenwald, 2006). L'article de Sylvain Broccolichi et Rémi Sinthon dans ce dossier souligne également les évolutions différentielles de réussite scolaire selon les milieux sociaux et leurs conséquences sur l'orientation. En outre, les enquêtes internationales $\mathrm{PISA}^{2}$ ont montré qu'en France, la dispersion des performances est importante et que la relation entre réussite et origine sociale est plus marquée que dans les autres pays développés (Baudelot \& Establet, 2009).

Ces études montrent que le poids de l'origine sociale sur la réussite d'un élève est deux fois plus fort en France qu'en Finlande ou au Japon, par exemple. Les rapports soulignent que la France se trouve dans la situation la plus défavorable parmi les pays de l'OCDE du point de vue de l'équité scolaire. Elle est le pays où l'écart des résultats entre les élèves de statuts "favorisés " et "défavorisés " est le plus important (Fumel, Keskpaik \& Girard, 2010). Entre 2000 et 2009, la proportion d'élèves français aux niveaux les plus faibles s'accroît alors que la pro- 
portion de bons élèves reste stable. De plus, le lien entre la performance d'un élève et le statut économique, social et culturel de ses parents reste très fort et s'est légèrement accentué entre les deux dernières enquêtes (PISA 2006 et PISA 2009).

Les évaluations nationales apportent les mêmes constats alarmants. Les écarts se creusent entre les résultats des élèves les meilleurs et les résultats des moins bons. En 2007, la direction de l'évaluation, de la prospective et de la performance du ministère de l'Éducation nationale (DEPP) a repris une enquête faite en 1987, en lecture, calcul et orthographe en fin de CM2. On constate alors sur vingt ans une baisse significative des performances des élèves dans ces trois compétences. Mais cette baisse est plus prononcée pour les élèves les plus faibles et les élèves des milieux populaires. La DEPP aboutit à des conclusions comparables en fin de collège, avec la reprise en 2009 d'une évaluation des compétences générales des élèves passée en 2003 : une baisse du score moyen des élèves entre les deux enquêtes, avec une augmentation de la proportion des élèves les plus faibles, tendance qui touche particulièrement les élèves de l'éducation prioritaire. Près d'un tiers des élèves scolarisés dans les collèges de l'éducation prioritaire font partie des groupes les moins performants en 2009, contre un quart il y a six ans (Bourny, Bessonneau, Daussin et al., 2010).

Enfin les analyses spatialisées du champ éducatif ont renouvelé les recherches sur les inégalités sociales de scolarisation en montrant comment les configurations locales influent sur la production (ou la résorption) d'inégalités, par l'effet cumulatif de l'offre de formation, des phénomènes de ségrégation et de la concurrence entre établissements (Broccolichi, Ben Ayed \& Trancart, 2010). Les conditions de scolarisation peuvent ainsi accentuer les difficultés d'apprentissage. Or les disparités entre collèges dues aux caractéristiques scolaires et sociales de leurs élèves se sont accusées durant les années quatre-vingt-dix (Trancart, 1998) ; de plus, on a montré que les modalités de constitution des classes peuvent accentuer ces inégalités, en provoquant une ségrégation interne à l'établissement (Duru-Bellat \& Mingat, 1997). Les travaux de Ben Ayed, Broccolichi et Trancart ont établi que dans les agglomérations où la ségrégation est forte, les collèges sont le lieu d'un ensemble de phénomènes - évitement, stigmatisation, tensions internes, instabilité des professionnels - qui ont des effets sur les conditions d'apprentissage, les élèves des milieux populaires étant les plus exposés aux conséquences de ces situations défavorables.
Ces analyses sont d'autant plus pertinentes que de nombreux facteurs concourent à un accroissement de la ségrégation urbaine. Par exemple, dans l'agglomération parisienne, les effets de la crise économique aboutissent à ce que les quartiers les plus populaires concentrent des populations de plus en plus précaires, souvent immigrées (Préteceille, 2006, 2009). La carte scolaire se superpose ainsi à un tissu urbain déjà très fortement différencié socialement. Mais elle tend aussi à renforcer la ségrégation résidentielle, dans la mesure où le choix du collège constitue un déterminant non négligeable du choix résidentiel des parents. Une enquête réalisée dans l'académie de Bordeaux mettait en évidence les processus de concentration d'élèves en difficulté et issus de l'émigration, en particulier le rôle des stratégies d'évitement de certains collèges (Felouzis, Liot \& Perroton, 2005). Enfin les " émeutes " urbaines de novembre 2005 ont pu être analysées comme "l'expression d'un sentiment profond de relégation et de disqualification chez la jeunesse des quartiers populaires ", où les différences de contexte scolaire contribuent à renforcer un sentiment d'injustice (Lagrange \& Oberti, 2006).

Le projet de suppression de la carte scolaire et l'accroissement des choix scolaires dans la constitution des trajectoires scolaires (cf. l'introduction de ce dossier) s'inscrivent donc dans ce contexte où les inégalités sociales et territoriales de réussite scolaire sont marquées et ont même tendance à s'accentuer. Diversifier l'élite, tel est l'objectif affiché en permettant aux parents de choisir l'établissement scolaire de leurs enfants. C'est aussi le sens d'autres dispositifs mis en place récemment visant à extraire de leur milieu quelques élèves "méritants": par exemple les " internats d'excellence ", les classes préparant à l'accès à l'Institut d'études politiques, la classe pilote pour élèves d'origine sociale modeste au sein du prestigieux lycée parisien Henri-IV... Ces dispositifs sont présentés comme des moyens d'aider des élèves de quartiers populaires ayant de bons résultats à intégrer des établissements plus prestigieux, ce qui devrait permettre de diversifier le recrutement social de l'élite. Si cet objectif est atteint, ce sera sans aucun doute de façon très marginale, en permettant à quelques bons élèves de zones défavorisées d'accéder à des établissements moins dégradés ou à des filières prestigieuses. Mais ces mesures individuelles ne résolvent en rien les problèmes massifs de l'ensemble du système éducatif (comme les inégalités sociales d'apprentissage) et au contraire aggravent la situation des établissements les plus fragilisés en les vidant de leurs bons élèves. On peut 
également s'interroger avec Choukri Ben Ayed sur l'efficacité de ces mesures pour ceux-là même qui en bénéficient. En revanche, les défis soulevés par l'échec scolaire dans les milieux populaires ne sont pas relevés, il est donc urgent que les sociologues, mais aussi les didacticiens (et pourquoi pas ensemble comme l'ont proposé Samuel Johsua et Bernard Lahire, 1999), s'intéressent aux conditions d'une bonne transmission des savoirs dans différents contextes sociaux.

Françoise CEuvrard fr.oeuvrard@orange.fr

\section{NOTES}

1 Expression de Marianne Blanchard et Joanie Cayouette-Remblière dans l'introduction de ce dossier.

2 PISA (Programme for International Student Assessment) est une enquête menée tous les trois ans auprès de jeunes de 15 ans dans les pays membres de l'OCDE. Les tests portent sur la lecture, la culture mathématique et la culture scientifique.

\section{BIBLIOGRAPHIE}

BAUDELOT C. \& ESTABLET R. (2009). L'élitisme républicain. L'école française à l'épreuve des comparaisons internationales. Paris : Éd. du Seuil.

BOURNY G., BESSONNEAU P., DAUSSIN J.-M. \& KESKPAIK S. (2010). "L'évolution des compétences générales des élèves en fin de collège de 2003 à 2009 ». Note d'information, $\mathrm{n}^{\circ} 10.22$.

BROCCOLICHI S., BEN AYED C. \& TRANCART D. (2010). École : les pièges de la concurrence. Comprendre le déclin de l'école française. Paris : La Découverte.

CAILLE J.-P. \& ROSENWALD F. (2006). « Les inégalités de réussite à l'école élémentaire : construction et évolution ». In INSEE, France, portrait social. Paris : INSEE, p. 115-137.

DURU-BELLAT M. \& MINGAT A. (1997). « La constitution des classes de niveau dans les collèges : les effets pervers d'une pratique à visée égalisatrice ". Revue française de sociologie, vol. $38, \mathrm{n}^{\circ} 4$, p. 759-789.
FELOUZIS G., LIOT F. \& PERROTON J. (2005). L'apartheid scolaire. Enquête sur la ségrégation ethnique dans les collèges. Paris : Éd. du Seuil.

FUMEL S., KESKPAIK S. \& GIRARD J. (2010). « L'évolution des acquis des élèves de 15 ans en compréhension de l'écrit : premiers résultats de l'évaluation internationale PISA 2009 ». Note d'information, n 10.24.

JOHSUA S. \& LAHIRE B. (1999). "Pour une didactique sociologique ». Éducation et sociétés, n4, p. 29-56.

LAGRANGE H. \& OBERTI M. (2006). Émeutes urbaines et protestations. Paris : Presses de Sciences Po.

PRÉTECEILLE E. (2006). « La ségrégation sociale a-t-elle augmenté ? La métropole parisienne entre polarisation et mixité ». Sociétés contemporaines, $n^{\circ} 62$, p. 69-93.

PRÉTECEILLE E. (2009). « La ségrégation ethno-raciale dans la métropole parisienne ". Revue française de sociologie, vol. 50, $n^{\circ} 3$, p. 489-519.

TRANCART D. (1998). "L'évolution des disparités entre collèges publics ". Revue française de pédagogie, $\mathrm{n}^{\circ} 124$, p. 43-54. 
